\title{
Shift Operator for Nonabelian Lattice Current Algebra $^{\dagger}$
}

By

\author{
Ludvig FADDEEV* and Alexander Yu. VOLKOV**
}

\begin{abstract}
The shift operator for a quantum lattice current algebra associated with $\operatorname{sl}(2)$ is produced in the form of product of local factors. This gives a natural deformation of the Sugawara construction for discrete space-time.
\end{abstract}

\section{Foreword by One of the Authors (LDF)}

I am very glad to participate in the volume, dedicated to 40 years of RIMS. My first visit to RIMS, where I was invited by Professors M. Sato and H. Araki, was in 1984. I gave several lectures on the quantum inverse scattering method (QISM), which was then still in the state of infancy. Doctors M. Jimbo and T. Miwa were the most attentive listeners and I believe, that this encounter influenced their scientific activity afterwards. In the following years the theme around QISM was greatly developed both in Leningrad and Kyoto, which led to very strong contacts between both schools. This paper is a result of my work with Dr. A. Volkov on the quantum integrable models on discrete space-time. Its preliminary version exists in the arXiv database [FV96] and here we publish an edited variant.

Communicated by M. Kashiwara. Revised November 14, 2003.

2000 Mathematics Subject Classification(s): 81R10, 81R50, 81R60

Key words: discrete space-time, nonabelian current, evolution operator

${ }^{\dagger}$ This article is an invited contribution to a special issue of Publications of RIMS commemorating the fortieth anniversary of the founding of the Research Institute for Mathematical Sciences.

* Saint Petersburg Branch of the Steklov Mathematical Institute Fontanka 27, Saint Petersburg 191023, Russia

**Vrije Universiteit Brussel, Pleinlaan 2, 1050 Brussel, Belgium 


\section{Introduction}

The Current Algebra provides the chiral dynamical variables for a generic conformal field theory model called WZNW model. Its lattice analogue, due to Semenov-Tian-Shansky, proved useful for elucidating the quantum group structure in this model [AFSV]. In the subsequent papers [AFS, FG, BC, AFFS] some general properties of this algebra and its representations were discussed. However, these considerations covered kinematical aspects of the lattice model while such a basic dynamical object as the hamiltonian density remained unavailable. Here we address this problem making use of our experience in a simpler abelian case [FV93, V97a]. Following the general philosophy worked out in these papers we construct a spatial translation operator $W$ which simultaneously generates the temporal shift. We find $W$ to be a product of local factors over the lattice. This may be regarded as a multiplicative analogue of the Sugawara construction.

For simplicity we confine ourselves to the simplest case of the $s l(2)$ algebra. In Section 1 we recall the basic facts about the current algebra in its classical continuous form. Then we embed the Sugawara hamiltonian into the hierarchies of conservation laws of two major integrable models which are mKdV and NLS equations $[\mathrm{FT}]$. To make a smoother transition to the quantum case we present in Section 3 the classical lattice deformation of the current algebra. In particular, we produce relevant integrable hierarchies. The quantum case is treated in Section 4.

\section{$\S 1 . \quad$ Classical Model}

The generators $j^{a}(x)$ of current algebra are associated with a given simple Lie algebra $g$ with index $a$ labeling the linear basis in $g$ and the variable $x$ running through the unit circle. Let $f_{c}^{a b}$ and $K^{a b}$ be the structure constants and the Killing tensor of $g$. The defining Poisson bracket is

$$
\left\{j^{a}(x), j^{b}(y)\right\}=\gamma f_{c}^{a b} j^{c}(x) \delta(x-y)+\gamma K^{a b} \delta^{\prime}(x-y) .
$$

The real 'coupling constant' $\gamma$ is irrelevant in classical case but comes into play under quantization.

The hamiltonian

$$
H=\frac{1}{2 \gamma} \int_{0}^{2 \pi} K_{a b} j^{a}(x) j^{b}(x) d x
$$

leads to a free equation of motion

$$
\partial_{t} j^{a}(x)=\left\{H, j^{a}(x)\right\}=\partial_{x} j^{a}(x)
$$


which reflects the conformal invariance in hyperbolic language. The hamiltonian density

$$
T(x)=K_{a b} j^{a}(x) j^{b}(x)
$$

is quadratic in the generators and is often referred to as the Sugawara construction. In this paper we shall consider $g$ to be a real form $\operatorname{sl}(2, R)$ of the algebra $s l(2)$.

Thus, $a$ takes the values $3,+,-$ and all the functions $j^{a}$ are real. The Poisson bracket is given by

$$
\begin{aligned}
\left\{j^{3}(x), j^{3}(y)\right\} & =\gamma \delta^{\prime}(x-y) \\
\left\{j^{3}(x), j^{ \pm}(y)\right\} & = \pm \gamma j^{ \pm} \delta(x-y) \\
\left\{j^{+}(x), j^{-}(y)\right\} & =2 \gamma\left(j^{3} \delta(x-y)+\delta^{\prime}(x-y)\right) \\
\left\{j^{ \pm}(x), j^{ \pm}(y)\right\} & =0
\end{aligned}
$$

and

$$
T=\left(j^{3}\right)^{2}+j^{+} j^{-} .
$$

It is also useful to combine the currents into a 2 by 2 matrix

$$
J=\left(\begin{array}{cc}
j^{3} & j^{-} \\
j^{+} & -j^{3}
\end{array}\right) .
$$

\section{§2. Separation of Variables and Yang-Baxterization}

The above bracket and hamiltonian allow for separation of variables. Indeed, one may put the matrix $J$ into the form

$$
J=\Omega\left(\begin{array}{c}
0 p \\
q 0
\end{array}\right) \Omega^{-1}+\partial_{x} \Omega \Omega^{-1}
$$

with a diagonal matrix $\Omega$ solving the equation

$$
\partial_{x} \Omega=j^{3} \sigma \Omega, \quad \sigma=\left(\begin{array}{cc}
1 & 0 \\
0 & -1
\end{array}\right)
$$

The Poisson bracket for the new set of dynamical variables $j \equiv j^{3}, p, q$ proves to be

$$
\begin{aligned}
& \{p(x), p(y)\}=-2 \gamma \operatorname{sign}(x-y) p(x) p(y) \\
& \{q(x), q(y)\}=-2 \gamma \operatorname{sign}(x-y) q(x) q(y) \\
& \{p(x), q(y)\}=2 \gamma\left(\operatorname{sign}(x-y) p(x) q(y)+\delta^{\prime}(x-y)\right) \\
& \{j(x), j(y)\}=\gamma \delta^{\prime}(x-y) \\
& \{j(x), p(y)\}=\{j(x), q(y)\}=0 .
\end{aligned}
$$


while the hamiltonian density becomes

$$
T=j^{2}+p q
$$

Thus, the pair $p, q$ completely separates from $j$. The $p-q$ bracket is known to belong to the hierarchy of Poisson structures associated with the NLS equation while the density $p q$ is a member (the momentum density) of the corresponding family of densities of local conservation laws $[\mathrm{FT}]$. On the other hand, the $j$-bracket and the density $j^{2}$ come from the hierarchy of the mKdV equation. Thus, we see where $s l(2)$ current algebra and Sugawara hamiltonian fit into the general pattern of Soliton Theory:

$$
H_{\mathrm{WZNW}}=P_{\mathrm{mKdV}}+P_{\mathrm{NLS}} \cdot
$$

This will prove useful for our approach to quantization.

The lattice formalism for the mKdV part, which is nothing but the abelian current algebra, was already developed in [FV93, V92, V97a]. In this paper we perform a similar treatment of the NLS part.

In Soliton Theory the densities of conservation laws come from the asymptotic expansion of the trace of the monodromy matrix of the auxiliary linear problem. For the NLS equation this problem reads

$$
\left(\partial_{x}+\left(\begin{array}{c}
0 p \\
q
\end{array}\right)+\lambda \sigma\right) \Psi=0 .
$$

The matrix $\Omega$ being diagonal, this auxiliary problem is gauge equivalent to

$$
\left(\partial_{x}+J+\lambda \sigma\right) \Psi=0 .
$$

Thus, we see that NLS part $P_{\text {NLS }}$ of the Sugawara hamiltonian is provided by 'Yang-Baxterization' of the current

$$
J \rightsquigarrow J+\lambda \sigma .
$$

In the next Section we shall do the same on the lattice.

\section{$\S 3 . \quad$ Lattice Model}

We discretize the circle introducing the spacial variable taking integer values running from 1 to $N$. The real dynamical variables will be denoted by $\alpha_{n+\frac{1}{2}}, \beta_{n}$ with integer $n$; it is understood that

$$
\begin{aligned}
\alpha_{n+N+\frac{1}{2}} & =\alpha_{n+\frac{1}{2}} \\
\beta_{n+N} & =\beta_{n} .
\end{aligned}
$$


One may say that integers label vertices while half-integers stand for edges. Or vice versa. One reason for using different notations for dynamical variables with integer and half-integer subscripts is mere convenience which becomes evident when the Poisson bracket is displayed:

$$
\begin{aligned}
\left\{\alpha_{n-\frac{1}{2}}, \alpha_{n+\frac{1}{2}}\right\} & =-2 \gamma \alpha_{n-\frac{1}{2}} \alpha_{n+\frac{1}{2}} \\
\left\{\alpha_{n-\frac{1}{2}}, \beta_{n}\right\} & =-2 \gamma \alpha_{n-\frac{1}{2}} \beta_{n} \\
\left\{\beta_{n}, \alpha_{n+\frac{1}{2}}\right\} & =-2 \gamma \beta_{n} \alpha_{n+\frac{1}{2}} \\
\left\{\beta_{n-1}, \beta_{n}\right\} & =2 \gamma \alpha_{n-\frac{1}{2}} .
\end{aligned}
$$

All brackets not listed are zero. It is clear that every variable has nontrivial brackets only with the two neighbours in either direction.

One can recognise here the so called Flaschka variables for the Toda model. However, the hierarchy we will deal with is different from that of the Toda equations.

To see what this lot has to do with the Current Algebra we arrange dynamical variables in two matrices

$$
\begin{aligned}
& B_{2 n}=\left(\begin{array}{cc}
\alpha_{2 n+\frac{1}{2}}^{-\frac{1}{2}} & 0 \\
0 & \alpha_{2 n+\frac{1}{2}}^{\frac{1}{2}}
\end{array}\right)\left(\begin{array}{cc}
1 \beta_{2 n} \\
0 & 1
\end{array}\right) \\
& C_{2 n-1}=\left(\begin{array}{cc}
\alpha_{2 n-\frac{1}{2}}^{\frac{1}{2}} & 0 \\
0 & \alpha_{2 n-\frac{1}{2}}^{-\frac{1}{2}}
\end{array}\right)\left(\begin{array}{cc}
1 & 0 \\
\beta_{2 n-1} & 1
\end{array}\right) .
\end{aligned}
$$

The Poisson relations for them

$$
\begin{aligned}
\left\{\stackrel{1}{B}_{2 n}, \stackrel{2}{B}_{2 n}\right\} & =\gamma\left[r_{12}, \stackrel{1}{B}_{2 n} \stackrel{2}{B}_{2 n}\right] \\
\left\{\stackrel{1}{C}_{2 n-1}, \stackrel{2}{C}_{2 n-1}\right\} & =\gamma\left[r_{21}, \stackrel{1}{C}_{2 n-1} \stackrel{2}{C}_{2 n-1}\right] \\
\left\{\stackrel{1}{B}_{2 n}, \stackrel{2}{C}_{2 n-1}\right\} & =\gamma \stackrel{1}{B}_{2 n} r_{12} \stackrel{2}{C}_{2 n-1} \\
\left\{\stackrel{1}{C}_{2 n+1}, \stackrel{2}{B}_{2 n}\right\} & =\gamma \stackrel{1}{C}_{2 n+1} r_{21} \stackrel{2}{B}_{2 n}
\end{aligned}
$$

employ the major ingredient of $q$-deformations, namely the classical $r$-matrices

$$
\begin{aligned}
& r_{12}=\left(\begin{array}{cccc}
\frac{1}{2} & 0 & 0 & 0 \\
0 & -\frac{1}{2} & 2 & 0 \\
0 & 0 & -\frac{1}{2} & 0 \\
0 & 0 & 0 & \frac{1}{2}
\end{array}\right) \\
& r_{21}=P_{12} r_{12} P_{12},
\end{aligned}
$$

where $P$ is a permutation. 
The product

$$
J_{n}=B_{2 n} C_{2 n-1}
$$

satisfies the Poisson brackets

$$
\begin{aligned}
\left\{\stackrel{1}{J}_{n}, \stackrel{2}{J}_{n}\right\} & =\gamma\left(r_{12} \stackrel{1}{J_{n}} \stackrel{2}{J_{n}}-\stackrel{1}{J_{n}} \stackrel{2}{J}_{n} r_{21}\right) \\
\left\{\stackrel{1}{J_{n+1}}, \stackrel{2}{J}_{n}\right\} & =\gamma \stackrel{1}{J_{n+1}} r_{21} \stackrel{2}{J_{n}}
\end{aligned}
$$

which turn into the Current Algebra in the continuum limit

$$
J_{n} \sim I+\Delta J(x) .
$$

This is what usually is called the Lattice Current Algebra. However, it is not clear whether one gains anything reducing $B$ - $C$-algebra to the $J$-one. This time we prefer to deal with somewhat more transparent $B$ - $C$-algebra but we could do with the $J$-one instead.

To produce relevant conservation laws we introduce the transfer-matrix

$$
t(\omega)=\operatorname{tr} \prod_{n}^{\leftarrow} \xi^{\sigma_{3}} B_{2 n} \eta^{-\sigma_{3}} C_{2 n-1}
$$

with spectral parameter' $\omega$ entering in $\xi$ and $\eta$ in such a way that

$$
\begin{aligned}
\xi^{2}+\eta^{2} & =2 \\
\frac{\xi}{\eta} & =\omega .
\end{aligned}
$$

It turns out that

(i) $t(\omega)$ is a Poisson commuting family:

$$
\left\{t(\omega), t\left(\omega^{\prime}\right)\right\}=0,
$$

(ii) in the continuous limit it turns into the trace of monodromy matrix of the continuous auxiliary linear problem of Section 1 provided

$$
\omega \sim 1+\Delta \lambda
$$

(iii) it is a power series in $\omega$

$$
t(\omega)=\sum_{-N / 2}^{N / 2} h_{k} \omega^{2 k}
$$


with

$$
\begin{aligned}
h_{N / 2} & =\prod_{n}\left(2+\frac{\beta_{2 n+1} \beta_{2 n}}{\alpha_{2 n+\frac{1}{2}}}\right) \\
h_{-N / 2} & =\prod_{n}\left(2+\frac{\beta_{2 n} \beta_{2 n-1}}{\alpha_{2 n-\frac{1}{2}}}\right) .
\end{aligned}
$$

(ii) is obvious, (iii) is almost so, (i) can be verified along the guidelines of [FM]. We shall not go into further details because the model in question actually belongs to the same hierarchy as the Ablowitz-Ladik's model [SV].

The local factors, entering the expressions above, coincide with the product of the offdiagonal elements of matrix $J_{n}$. Thus in the continuous limit we have

$$
H=\log h_{N / 2}+\log h_{-N / 2}-N \log 2=\frac{\Delta}{2} \int_{0}^{2 \pi} J^{+} J^{-} d x=\Delta P_{\mathrm{NLS}}
$$

as should be expected.

We have obtained the hamiltonian of the classical lattice model which plays the role of the NLS part of the Sugawara construction for the lattice current algebra. The corresponding $\mathrm{mKdV}$ part can be found in [V92]. However, the equations of motion produced by these hamiltonians are quite complicated and turn into simple free equations only in the continuous limit. It was realized in [FV93] that the discrete time equation

$$
J_{n}(t+\Delta)=J_{n+1}(t)
$$

is a better option. In other words, the discretizing of space should be accompanied by the discretizing of time. The last equation is especially transparent in the quantum theory where the spacial shift operator $W$ such that

$$
W^{-1} J_{n} W=J_{n+1}
$$

is taken to define the time shift as well

$$
\begin{aligned}
J_{n}(t+\Delta) & =W^{-1} J_{n}(t) W \\
J_{n}(0) & =J_{n} .
\end{aligned}
$$

The matrix elements of $J_{n}$ depend on the variables $\alpha_{2 n \pm \frac{1}{2}}, \beta_{2 n}, \beta_{2 n-1}$, so that the shift $n \rightarrow n+1$ corresponds for the shift for $\alpha, \beta$ on two lattice points

$$
\begin{gathered}
\alpha_{n-\frac{1}{2}} W=W \alpha_{n+\frac{3}{2}} \\
\beta_{n-1} W=W \beta_{n+1}
\end{gathered}
$$


One can say, that the site on the chain for $J_{n}$ consists of two sites of the chain for $\alpha-\beta$ variables.

We shall find operator $W$ in the next Section. The expression for the classical lattice hamiltonian will prove to be a useful hint in our search.

\section{§4. Shift Operator}

The quantum lattice current algebra inherits the notation $\alpha$ - $\beta$ for generators together with the way they are enumerated while the Poisson relations turn into their most natural quantum counterparts

$$
\begin{aligned}
\alpha_{n+\frac{1}{2}} \alpha_{n-\frac{1}{2}} & =q^{2} \alpha_{n-\frac{1}{2}} \alpha_{n+\frac{1}{2}} \\
\beta_{n} \alpha_{n-\frac{1}{2}} & =q^{2} \alpha_{n-\frac{1}{2}} \beta_{n} \\
\alpha_{n+\frac{1}{2}} \beta_{n} & =q^{2} \beta_{n} \alpha_{n+\frac{1}{2}} \\
{\left[\beta_{n-1}, \beta_{n}\right] } & =\left(q-q^{-1}\right) \alpha_{n-\frac{1}{2}},
\end{aligned}
$$

with the deformation parameter $q$ combining the coupling constant $\gamma$ and the Planck constant $\hbar$ in the usual way

$$
q=e^{i \hbar \gamma}
$$

The consistency of these commutation relations becomes more apparent as soon as one rewrites them in $R$-matrix form

$$
\begin{aligned}
& R_{12} \stackrel{1}{B} \underset{2 n}{2} \stackrel{2}{B}_{2 n}=\stackrel{2}{B}_{2 n} \stackrel{1}{B}{ }_{2 n} R_{12} \\
& R_{21} \stackrel{1}{C}_{2 n-1} \stackrel{2}{C}_{2 n-1}=\stackrel{2}{C}_{2 n-1} \stackrel{1}{C}_{2 n-1} R_{21} \\
& \stackrel{2}{C}_{2 n-1} \stackrel{1}{B}_{2 n}=\stackrel{1}{B}_{2 n} R_{12} \stackrel{2}{C}_{2 n-1} \\
& \stackrel{2}{B}_{2 n} \stackrel{1}{C}_{2 n+1}=\stackrel{1}{C}_{2 n+1} R_{21} \stackrel{2}{B}_{2 n}
\end{aligned}
$$

where matrices $B, C$ are built of $\alpha, \beta$ 's in literally the same way as in the classical case of Section 2. The $R$-matrix involved is the $\operatorname{sl}(2)$ one

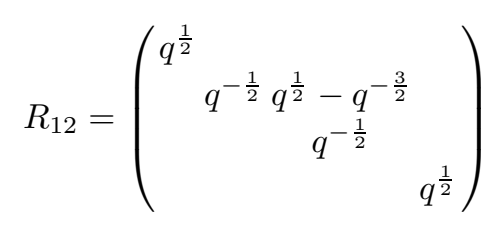

and it is needless to say that the associativity of the $B$ - $C$-algebra is due to the Yang-Baxter equation

$$
R_{12} R_{13} R_{23}=R_{23} R_{13} R_{12}
$$

fulfilled by $R$. 
The way variables separate in the continuous model and the belief that integrals of local densities on the lattice turn into products of local factors suggest that the shift operator $W$ should decompose into a product of two commuting factors

$$
W=U V=V U
$$

depending separately on mKDV and NLS variables

$$
\begin{gathered}
\xi_{n}=\alpha_{n+\frac{1}{2}} \alpha_{n-\frac{1}{2}}^{-1} \\
t_{n-\frac{1}{2}}=q+q^{2} \beta_{n} \alpha_{n-\frac{1}{2}}^{-1} \beta_{n-1}
\end{gathered}
$$

as follows

$$
U=\prod^{\leftarrow} \theta_{n}, \quad V=\overleftarrow{\prod} \sigma_{n}
$$

where

$$
\theta_{n}=\theta\left(q \xi_{n}\right), \quad \sigma_{n}=\sigma\left(t_{n-\frac{1}{2}}\right)
$$

with appropriate functions $\theta$ and $\sigma$. The arrow shows that local numbers increase from right to left. The functions $\theta(\xi)$ and $\sigma(t)$ are taken from our previous papers e.g. [FKV]. They satisfy the functional equations

$$
\frac{\theta(q \xi)}{\theta\left(q^{-1} \xi\right)}=-\xi
$$

and

$$
\frac{\sigma(q t)}{\sigma\left(q^{-1} t\right)}=\frac{1}{1+t} .
$$

It was shown in [FV93] that $\theta_{n}$ are generators of the Braid group

$$
\theta_{n+1} \theta_{n} \theta_{n+1}=\theta_{n} \theta_{n+1} \theta_{n}
$$

and the cyclic product of $2 N-1$ generators does not depend on the point of departure

$$
\theta_{2 N} \ldots \theta_{2}=\theta_{2 N-1} \ldots \theta_{1}=\theta_{2 N-2} \ldots \theta_{1} \theta_{N}=\ldots,
$$

if we impose an appropriate condition on the central elements of $\alpha$-algebra. The product defining operator $U$ is just this one.

The generators $\sigma_{n}$ satisfy the relations which were first derived by one of the authors $(\mathrm{AYuV}$, see [V97b])

$$
\begin{aligned}
\sigma_{n+1} \sigma_{n-1} \sigma_{n} \sigma_{n+1} & =\sigma_{n-1} \sigma_{n+1} \sigma_{n} \\
\sigma_{n} \sigma_{n-1} \sigma_{n+1} & =\sigma_{n-1} \sigma_{n} \sigma_{n+1} \sigma_{n-1}
\end{aligned}
$$


We conjecture, that the cyclic product of $\sigma_{n}$ shortened by two factors, also does not depend on the end point

$$
\sigma_{2 N} \sigma_{2 N-1} \ldots \sigma_{3}=\sigma_{2 N-1} \ldots \sigma_{2}=\sigma_{2 N-2} \ldots \sigma_{1}=\ldots
$$

The derivation, as in the case of $\theta_{n}$, should use the structure of the set of central elements in $\alpha-\beta$ algebra and is not done yet. So what follows depends heavily on the validity of this conjecture.

In a more weak formulation the conjecture states the existence of the cyclic product of factors $\left(\sigma_{2 N} \ldots \sigma_{1}\right)_{\text {cycl }}$ with the following properties:

1. It does not depend on the point of departure

$$
\left(\sigma_{2 N} \ldots \sigma_{1}\right)_{\mathrm{cycl}}=\left(\sigma_{k} \ldots \sigma_{1} \sigma_{2 N} \ldots \sigma_{k+1}\right)_{\mathrm{cycl}} .
$$

2. For a chosen variant only dependence on variables $t_{k}$ near the beginning and the end of the chain is modified, so that factors in the bulk of the chain stay intact.

This allows to reduce check of the property of shift to local calculations.

Let us stress, that the RHS of the functional equation for $\sigma(t)$ comes directly from the density of the classical lattice hamiltonian

$$
\frac{1}{1+t} \sim \frac{1}{2+\beta \beta / \alpha}
$$

This correspondence principle plays a major role in the detailed study of the classical limit which will be presented elsewhere.

As we said, one could do with the $J$-picture from the very beginning. This would eventually lead to the following decomposition

$$
W=\prod^{\leftarrow} \sigma\left(t_{2 n+\frac{1}{2}}\right) \theta\left(q \alpha_{2 n+\frac{1}{2}} \alpha_{2 n-\frac{1}{2}}^{-1}\right) \sigma\left(t_{2 n-\frac{1}{2}}\right)
$$

for the $J$-shifting operator

$$
J_{n} W=W J_{n+1} .
$$

This reduction is based on the factorization of the algebra of abelian currents $\alpha_{n-\frac{1}{2}}$ into two commuting ones, generated by

$$
k_{n}=\frac{\alpha_{2 n+\frac{1}{2}}}{\alpha_{2 n-\frac{1}{2}}} \quad \text { and } \quad l_{n}=\alpha_{2 n+\frac{3}{2}} \alpha_{2 n+\frac{1}{2}} .
$$

Now we can turn to the derivation. Due to the cyclicity and locality of expressions for $U$ and $V$ the equations

$$
\alpha_{n-\frac{1}{2}} W=W \alpha_{n+\frac{3}{2}}
$$




$$
\beta_{n-1} W=W \beta_{n+1}
$$

easily reduces to

$$
\begin{aligned}
& \alpha_{n-\frac{1}{2}} \theta\left(q \alpha_{n+\frac{3}{2}} \alpha_{n+\frac{1}{2}}^{-1}\right) \theta\left(q \alpha_{n+\frac{1}{2}} \alpha_{n-\frac{1}{2}}^{-1}\right) \\
& \quad=\theta\left(q \alpha_{n+\frac{3}{2}} \alpha_{n+\frac{1}{2}}^{-1}\right) \theta\left(q \alpha_{n+\frac{1}{2}} \alpha_{n-\frac{1}{2}}^{-1}\right) \alpha_{n+\frac{3}{2}} .
\end{aligned}
$$

and

$$
\begin{aligned}
& \beta_{n-1} \sigma\left(t_{n+\frac{1}{2}}\right) \theta\left(q \alpha_{n+\frac{1}{2}} \alpha_{n-\frac{1}{2}}^{-1}\right) \sigma\left(t_{n-\frac{1}{2}}\right) \\
& =\sigma\left(t_{n+\frac{1}{2}}\right) \theta\left(q \alpha_{n+\frac{1}{2}} \alpha_{n-\frac{1}{2}}^{-1}\right) \sigma\left(t_{n-\frac{1}{2}}\right) \beta_{n+1} .
\end{aligned}
$$

The form of the functional equations suggests, that any computation must be based on the Weyl-type relations $x y=q^{2} y x$.

Since any two of the $\alpha$ 's either commute or make a Weyl pair, the first translation comes easy:

$$
\begin{aligned}
& \alpha_{n-\frac{1}{2}} \theta\left(q \alpha_{n+\frac{3}{2}} \alpha_{n+\frac{1}{2}}^{-1}\right) \theta\left(q \alpha_{n+\frac{1}{2}} \alpha_{n-\frac{1}{2}}^{-1}\right) \\
& \quad=-q^{2} \theta\left(q \alpha_{n+\frac{3}{2}} \alpha_{n+\frac{1}{2}}^{-1}\right) \alpha_{n+\frac{3}{2}} \alpha_{n+\frac{1}{2}}^{-1} \alpha_{n-\frac{1}{2}} \theta\left(q \alpha_{n+\frac{1}{2}} \alpha_{n-\frac{1}{2}}^{-1}\right) \\
& \quad=q^{4} \theta\left(q \alpha_{n+\frac{3}{2}} \alpha_{n+\frac{1}{2}}^{-1}\right) \theta\left(q \alpha_{n+\frac{1}{2}} \alpha_{n-\frac{1}{2}}^{-1}\right) \alpha_{n+\frac{1}{2}} \alpha_{n-\frac{1}{2}}^{-1} \alpha_{n+\frac{3}{2}} \alpha_{n+\frac{1}{2}}^{-1} \alpha_{n-\frac{1}{2}} \\
& \quad=\theta\left(q \alpha_{n+\frac{3}{2}} \alpha_{n+\frac{1}{2}}^{-1}\right) \theta\left(q \alpha_{n+\frac{1}{2}} \alpha_{n-\frac{1}{2}}^{-1}\right) \alpha_{n+\frac{3}{2}} .
\end{aligned}
$$

The second one is more tricky. We cannot pull $\beta_{n-1}$ through $\sigma\left(t_{n+\frac{1}{2}}\right)$ straight away because $\beta_{n-1}$ and $t_{n+\frac{1}{2}}$ neither commute nor make a Weyl pair. Nevertheless, we have a good supply of operators making 'good' pairs with both $t_{n-\frac{1}{2}}$ and $t_{n+\frac{1}{2}}$ which, by the way, between themselves are a $q$-oscillator ${ }^{1}$

$$
q t_{n+\frac{1}{2}} t_{n-\frac{1}{2}}-q^{-1} t_{n-\frac{1}{2}} t_{n+\frac{1}{2}}=q-q^{-1}
$$

Among them are:

(i) all the $\alpha$ 's

$$
[\alpha, t]=0,
$$

\footnotetext{
${ }^{1}$ The remaining nontrivial commutation relations governing the algebra of $t$ 's, those for the neighbours twice removed, seldom participate in computations. Their role may be seen in taking care of the associativity of the algebra. Anyway, their explicit form can be found in [V92]. This algebra is sometimes referred to as the Lattice Virasoro Algebra for in a certain continuous limit, different from the one of the present paper, it turns into the Virasoro algebra with a nonzero central charge.
} 
(ii) the $\beta$ which is 'between' them

$$
\begin{aligned}
& t_{n-\frac{1}{2}} \beta_{n}=q^{2} \beta_{n} t_{n-\frac{1}{2}} \\
& \beta_{n} t_{n+\frac{1}{2}}=q^{2} t_{n+\frac{1}{2}} \beta_{n}
\end{aligned}
$$

(iii) another operator $c_{n}=q\left(t_{n+\frac{1}{2}} t_{n-\frac{1}{2}}-1\right)$

$$
\begin{aligned}
& t_{n-\frac{1}{2}} c_{n}=q^{2} c_{n} t_{n-\frac{1}{2}} \\
& c_{n} t_{n+\frac{1}{2}}=q^{2} t_{n+\frac{1}{2}} c_{n}
\end{aligned}
$$

which is a familiar satellite of $q$-oscillators.

So, we express $\beta_{n-1}$ via 'good' operators

$$
\beta_{n-1}=q t_{n+\frac{1}{2}}^{-1} c_{n} \beta_{n}^{-1} \alpha_{n-\frac{1}{2}}+q^{2} t_{n+\frac{1}{2}}^{-1} \beta_{n}^{-1} \alpha_{n-\frac{1}{2}}-q \beta_{n}^{-1} \alpha_{n-\frac{1}{2}}
$$

and get

$$
\begin{aligned}
& \beta_{n-1} \sigma\left(t_{n+\frac{1}{2}}\right) \\
= & \sigma\left(t_{n+\frac{1}{2}}\right)\left(q t_{n+\frac{1}{2}}^{-1} c_{n} \beta_{n}^{-1} \alpha_{n-\frac{1}{2}}+\left(1+q^{-1} t_{n+\frac{1}{2}}\right)\left(q^{2} t_{n+\frac{1}{2}}^{-1} \beta_{n}^{-1} \alpha_{n-\frac{1}{2}}-q \beta_{n}^{-1} \alpha_{n-\frac{1}{2}}\right)\right) \\
= & \left.\sigma\left(t_{n+\frac{1}{2}}\right)\left(\left(\beta_{n}^{-1} t_{n-\frac{1}{2}}-t_{n+\frac{1}{2}} \beta_{n}^{-1}\right) \alpha_{n-\frac{1}{2}}\right)\right) .
\end{aligned}
$$

Similarly,

$$
\sigma\left(t_{n-\frac{1}{2}}\right) \beta_{n+1}=\left(\alpha_{n+\frac{1}{2}}\left(-\beta_{n}^{-1} t_{n-\frac{1}{2}}+t_{n+\frac{1}{2}} \beta_{n}^{-1}\right)\right) \sigma\left(t_{n-\frac{1}{2}}\right) .
$$

The rest

$$
\begin{aligned}
& \left.\left(\left(\beta_{n}^{-1} t_{n-\frac{1}{2}}-t_{n+\frac{1}{2}} \beta_{n}^{-1}\right) \alpha_{n-\frac{1}{2}}\right)\right) \theta\left(q \alpha_{n+\frac{1}{2}} \alpha_{n-\frac{1}{2}}^{-1}\right) \\
& \quad=\theta\left(q \alpha_{n+\frac{1}{2}} \alpha_{n-\frac{1}{2}}^{-1}\right)\left(\alpha_{n+\frac{1}{2}}\left(-\beta_{n}^{-1} t_{n-\frac{1}{2}}+t_{n+\frac{1}{2}} \beta_{n}^{-1}\right)\right)
\end{aligned}
$$

is a variation on the same theme.

This completes the derivation and our paper. We hope to prove the conjecture in the near future. 


\section{Acknowledgements}

We are grateful to A. Alekseev and M. Semenov-Tian-Shansky for stimulating discussions. The work was partly supported by the RFBR grant 02-0100085 .

\section{References}

[AFFS] Alekseev, A., Faddeev, L., Fröhlich, J. and Shomerus, V., Representation Theory of Lattice Current Algebras, Comm. Math. Phys., 191 (1998), 31-60.

[AFS] Alekseev, A., Faddeev, L. and Semenov-Tian-Shansky, M., Hidden Quantum Groups Inside Kac-Moody Algebra, Comm. Math. Phys., 149 (1992), 335-345.

[AFSV] Alekseev, A., Faddeev, L., Semenov-Tian-Shansky, M. and Volkov, A., The unravalling of the quantum group structure in the WZNW theory, Preprint CERNTH-5981/91.

[BC] Belov, A. and Chaltikian, K., Lattice Virasoro from Lattice Kac-Moody, Phys. Lett. $B, 317$ (1993), 73-77.

[F] Faddeev, L., Current-Like Variables in Massive and Massless Integrable Models, hep-th/9408041.

[FG] Falceto, F. and Gawedzky, K., Lattice Wess-Zumino-Witten Model and Quantum groups, J. Geom. Phys., 11 (1993), 251-279.

[FKV] Faddeev, L., Kashaev R. and Volkov, A., Strongly Coupled Quantum Discrete Liouville Theory. 1. Algebraic approach and duality, Comm. Math. Phys., 219 (2001), 199-219.

[FM] Freidel, L. and Maillet, J. M., On Classical and Quantum Integrable Field Theories Associated to Kac-Moody Current Algebras, Phys. Lett. B, 263 (1991), 403-410.

[FT] Faddeev, L. and Takhtajan, L., Hamiltonian methods in the theory of solitons, Springer, Berlin, 1987.

[FV93] Faddeev, L. and Volkov, A., Abelian Current Algebra and the Virasoro Algebra on the Lattice, Phys. Lett. B, 315 (1993), 311-318.

[FV94] - Hirota Equation as an Example of Integrable Symplecttic Map, Lett. Math. Phys., 32 (1994), 125-135.

[FV95] - Yang-Baxterization of quantum dilogarithm, Zap. Nauchn. Semin., POMI, 224 (1995).

[FV96] Shift Operator for Nonabelian Lattice Current Algebra, hep-th/9606088, RIMS, Kyoto Univ., 40 (2004), 1113-1125.

[SV] Sklyanin, E. and Volkov, A., Yu., unpublished.

[V92] Volkov, A., Quantum Volterra model, Phys. Lett. A, 167 (1992), 345.

[V97a] , Quantum Lattice KDV Equation, Lett. Math. Phys., 39 (1997), 313-329, hep-th/9509024.

[V97b] , Beyond the "Pentagon Identity", Lett. Math. Phys., 39 (1997), 393-397. 